Verlag Dr. Otto Schmidt KG, Postfach 5110 26, 50946 Köln · Gustav-Heinemann-Ufer 58 $50968 \mathrm{Köln} \cdot$ Tel. 02 21/9 37 38-9 97 (Vertrieb/Abonnementsverwaltung), Fax 02 21/9 37 38-9 43 (Vertrieb/Abonnementsverwaltung). Erfüllungsort und Gerichtsstand ist Köln.

Redaktion: RAin Stefanie Fuchs-Galilea, LL.M. (verantwortlich), Anschrift des Verlags · RA Georg Wallraf, Kerpen · RAin Christine Libor, Düsseldorf

Redaktionssekretariat: Sandra Roeseler, Tel. 02 21/9 37 38-1 69, Fax 02 21/9 37 38-9 06, E-Mail: afp@otto-schmidt.de, Internet: http://www.afp-medienrecht.de

Unter Mitwirkung von: RA Dr. Simon Assion, Frankfurt/M. · RA Dr. Stefan Engels, Hamburg · RA Dominik Eickemeier, Köln · Dr. Thomas Haug, LL.M., Frankfurt/M. · RA Dr. Ruben A. Hofmann, Köln · RA Dr. Martin Jäger, Düsseldorf · RA Prof. Dr. Reinhard Ricker, Frankfurt/M. · RA Dr. Jörg Witting, Düsseldorf

\section{Herstellung: Karina Hack}

Anzeigenverkauf: sales friendly Verlagsdienstleistungen, Pfaffenweg 15, 53227 Bonn Tel. 02 28/9 78 98-0, Fax 02 28/9 78 98-20, E-Mail: media@sales-friendly.de · gültig ist die Preisliste Nr. 48 v. 1.1.2018.

Druck: rewi Druckhaus, Reiner Winters GmbH, Wiesenstr. 11, 57537 Wissen, druckhaus@rewi.de,www.rewi.de.

Erscheinungsweise: Sechsmal jährlich zum Monatsende Februar, April, Juni, August, Oktober, Dezember

Bezugspreis: Jahresabonnement inkl. App-Zugang 279,- $€$ (Print-Anteil 264,- $€^{*} / 0 n$ lineAnteil 14,- $\left.€^{\star \star}\right)$; für Mitglieder der Arbeitsgemeinschaft Geistiges Eigentum \& Medien im Deutschen Anwaltverein (AGEM) 239- - $€$ (Print-Anteil 225- - $€^{*} / 0$ nline-Anteil 14- $€^{* *}$ ): für Studenten, Referendare (fachbezogen) und Anwälte, deren Zulassung jünger ist als drei Jahre (gegen Nachweis) 99,- $€$ (Print-Anteil 94,- $€^{\star}$, Online-Anteil 5,- $€^{\star *}$ ); Einzelheft 50,60 €. Alle Preise verstehen sich inkl. gesetzlicher MwSt. *7 \% oder **19 \% sowie zzgl. Versandkosten. Die Rechnungsstellung erfolgt jährlich zu Beginn des Bezugszeitraumes für das aktuelle Kalenderjahr (ggf. anteilig). Bestellungen bei jeder Buchhandlung sowie beim Verlag. Kündigungstermin für das Abonnement sechs Wochen vor Jahresschluss.

ISSN: 0949-2100 (Print) · 2366-0945 (eJournal)

Urheber- und Verlagsrechte: Mit Annahme eines Manuskripts (Aufsatz, Bearbeitung Leitsatz, Blog-Text) geht für die Dauer von vier Jahren das räumlich unbeschränkte, allei- nige und uneingeschränkte (ausschließliche), danach das einfache Nutzungsrecht vom Autor auf den Verlag über, jeweils auch für Übersetzungen, Nachdrucke, Nachdruckgenehmigungen und die Kombination mit anderen Werken oder Teilen daraus. Soweit ein Beitrag zur Lern- und Erfolgskontrolle - auch im Rahmen des Fortbildungsnachweises für einen Fachanwalt gem. § 15 FAO - vorgesehen ist, erstreckt sich die Nutzungsrechtsübertragung auch auf die vom Autor hierzu formulierten Fragen und Antworten. Das Nutzungsrecht umfasst das Recht zur Vervielfältigung und Verbreitung in gedruckter Form. Zur eigenen Vermarktung, zur gemeinsamen Vermarktung mit einem Kooperationspartner, zur Vermarktung durch ein Unternehmen, an dem der Verlag überwiegend beteiligt ist, und/oder zur Vermarktung durch einen Dritten, den der Verlag hierzu berechtigt, umfasst das Nutzungsrecht ferner insbesondere die Befugnis zur vollständigen oder teilweisen Aufzeichnung in elektronischer Form, zur Programmierung, sonstigen Be- und Verarbeitung für eine elektronische Nutzung einschließlich Zusammenlegung mit anderen Werken zu einem elektronischen Produkt sowie Speicherung in eigenen oder fremden Datenverarbeitungsanlagen, in elektronischen Datenbanken und auf Datenträgern sowie zur Vervielfältigung, Verbreitung einschließlich der öffentlichen Wiedergabe und zur sonstigen Nutzung im Wege fotomechanischer, elektronischer und anderer Verfahren, insbesondere in elektronischen Offline- und Online-Datenbanken und -Diensten, im Wege von Wiedergabetechniken in körperlicher und/oder nichtkörperlicher Form, im Wege von allen Techniken der Datenübertragung sowie auf Datenträgern, z.B. CD-ROM, DVD, Stick und vergleichbaren Techniken. Der Autor versichert, über die urheberrechtlichen Nutzungsrechte an seinem Beitrag einschließlich aller Abbildungen allein verfügen zu können und keine Rechte Dritter zu verletzen. Dies gilt auch für Entscheidungen und deren Leitsätze, soweit sie vom Einsender redigiert bzw. erarbeitet wurden. Die Zeitschrift und alle veröffentlichten Beiträge und Abbildungen sind urheberrechtlich geschützt. Jede vom Urheberrechtsgesetz nicht ausdrücklich zugelassene Verwertung des Zeitschrifteninhalts bedarf einer vorherigen schriftlicher Zustimmung des Verlags. Das Zitieren von Rezensionen ist in vollem Umfang erlaubt.

Hinweise für Autoren und Einsender: Bitte senden Sie alle Manuskripte (als Datei pe E-Mail), zum Abdruck bestimmte Gerichtsentscheidungen und Leserbriefe unmittelbar an die Redaktion. Bei der Einsendung von Gerichtsentscheidungen sind wir für den Hinweis dankbar, ob sie rechtskräftig sind. Wird im Fall des Abdrucks eine Pauschalvergütung gezahlt, gilt sie für die Übertragung eines ggf. bestehenden Nutzungsrechts mit der Maßgabe, die Entscheidung auch in anderen Print- und elektronischen Produkten des Verlages veröffentlichen zu können.

\title{
Jetzt abonnieren!
}

\section{Diese Vorteile bietet Ihnen ein Abonnement:}

\author{
Alle zwei Monate die Zeitschrift - plus Beiträge zum Selbststudium \\ nach $§ 15$ FAO.
}

Zugriff auf das Beratermodul AfP - Medienrecht mit dem Archiv der Zeitschrift seit 2000 sowie Volltexte zu Gesetzen, Gerichtsentscheidungen und Verwaltungsanweisungen.

Mobile Nutzung über die Otto-Schmidt-Zeitschriften-App.

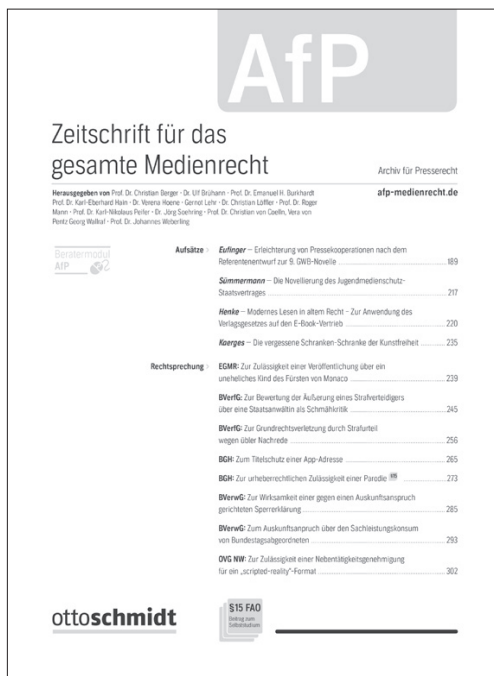

Das kostenlose Probe-Abo mit 1 Heft inkl. App und Beratermodul AfP - Medienrecht gibt's hier: www.otto-schmidt.de/zsafp oder telefonisch beim Aboservice-Telefon 0221 93738-997 